\title{
Impact of Local Anesthetics on Intrathecal Anesthesia
}

\author{
Carlos De La Paz Estrada* \\ Department of Anesthesiology and Reanimation,Provincial Pediatric Hospital, Cuba
}

*Corresponding author: Carlos De La Paz Estrada, Department of Anesthesiology and Reanimation, Cuba.

Received Date: March 16, 2020

Published Date: April 01, 2020

\section{Summary}

Of all regional techniques, subarachnoid block is the fastest, most predictable, and most reliable. It is a technique that has unique characteristics for use in ambulatory surgery: immediate initiation of anesthesia, predictable response according to the dose administered, and the possibility of adjusting the anesthetic duration by selecting the appropriate agent. Its main advantages are its easy realization, the quick establishment of the lock, its high effectiveness and reliability and its low toxicity. It is a simple technique to perform since anatomical references are usually easy to locate and the procedure is performed in a short time, even in obese patients. The quality of the blockage is especially evident in interventions of the lower extremities performed with tourniquet and in perineal surgery, thanks to its ability to achieve complete sacral anesthesia. It has low toxicity because the small doses of local anesthetic required eliminate the risk of systemic toxicity in case of inadvertent intravascular injection.

\section{Introduction}

Subarachnoid anesthesia was introduced into clinical practice during the last decade of the 19th century by Bier in Germany and Tuffier in France. The first spinal anesthesia in America is attributed to Tait and Caglieri in San Francisco California.

In 1947, Cecil Roe and Albert Wooley obtained permanent spastic and painful paraparesis after spinal anesthesia with hypobaric chinchocaine administered the same day and by the same anesthesiologist. The hospital and anesthesiologist were sued, and in 1953, the case dealt with multiple discussions in the English courts and finally produced a negative effect on the evolution of subarachnoid anesthesia, not only in the UK, but worldwide, delaying the development of this anesthetic modality. Noble, Moore et al, and Dripps and Vandam reviewed hundreds of patients and affirmed the concept that subarachnoid anesthesia is a safe procedure.

Due to what has been previously described, and the search for new local anesthetics (LA) were determining factors for many anesthesiologists to rediscover the benefits of this technique, however, post-traumatic headache (CPR) has been a determining factor in the low acceptance of this procedure anesthetic.

The appearance on the international market of improved spinal needles, and the availability of novel levoisomeric ALs and less toxic and long-acting adjuvant drugs, have made spinal anesthesia a procedure that today is used more frequently and safely.

Increasing the dose of an intrathecal AL increases the duration of anesthesia and the time required for recovery, and vice versa. The dose-response relationship allows us to select an LA appropriately, with or without an adjuvant drug, for a specific duration of spinal anesthesia [1-3].

\section{Lidocaine}

Lidocaine, synthesized in 1943 by Löfgren, was introduced to the clinic in 1947. This drug is by far the most widely used LA by all routes in regional anesthesia, including Bier's block. It is a fastonset amido-amide with an intermediate duration of action. Since the origin of outpatient surgery, lidocaine was the most popular AL in spinal anesthesia.

There is a tendency to abandon its routine use by the intrathecal route due to its neurotoxic power and the possibility of transient irritation syndrome of the posterior roots (ITRP), even with solutions diluted to $2.5 \%, 2 \%$ and $0.5 \%$, as well as cases of cauda equina. These data are still highly controversial, although numerous clinical investigations have shown that lidocaine is the most important factor in the development of ITRP, which can occur in up to $20 \%$ of outpatients. The lidocaine dose can be reduced 
to $30-15 \mathrm{mg}$, by adding low doses of opioids (fentanyl $12.5 \mu \mathrm{g}$ or sufentanil $10 \mu \mathrm{g}$ ) to facilitate early recovery in short perineal or pelvic procedures.

ITRP and cases of cauda equina attributed to intrathecal lidocaine led to other LAs being experimented with in this way in an attempt to replace lidocaine. The LAs of the pipecoloxilidide family (bupivacaine, ropivacaine, and mepivacaine) are the most recommended since their duration of action is sufficient for most outpatient procedures, and by reducing the doses, their duration and side effects have been reduced [4-6].

\section{Bupivacaine}

Bupivacaine, developed in 1957, is a racemic AL that contains two stereoisomers, $\mathrm{S}$ - and $\mathrm{R}+$, the latter being the one with the greatest potential for toxicity. It has been shown to be a suitable substitute for intrathecal lidocaine for outpatient procedures. The usual doses from 15 to $18 \mathrm{mg}$ can be decreased to $10 \mathrm{mg}$ to avoid urinary retention and delay in the time of home discharge. The almost null possibility that ITRP exists, favors the use of bupivacaine in this group of patients.

Bupivacaine $8 \mathrm{mg}$ 0.5\% hyperbaric vs. $8 \mathrm{mg}$ has been compared. sciatic-femoral block with mepivacaine, and it has been shown that peripheral blocks require more time to perform spinal anesthesia. Patients treated with spinal cord had a shorter block but took longer to urinate, without affecting discharge time. We can decrease the dose to $4 \mathrm{mg}$ of $0.25 \%$ bupivacaine, hyperbaric, thereby reducing the height of the sensory block and the duration of the motor block. A dose of $3 \mathrm{mg}$ of hyperbaric bupivacaine added with $10 \mu \mathrm{g}$ of fentanyl with a lateral technique for $10 \mathrm{~min}$ produces sufficient anesthesia for outpatient arthroscopies. The intrathecal injection of $9.7 \mathrm{mg}$ of isobaric bupivacaine acts 5 minutes earlier than the hyperbaric form, although at $15 \mathrm{~min}$ both have the same sensitive level and similar intensity of motor block, similar characteristics occur when $15 \mathrm{mg}$ of this LA are injected [7-9].

\section{Levobupivacaine}

Levobupivacaine is a long-acting levoisomer, with a clinical profile similar to the racemic mixture of bupivacaine, but with a lower spectrum of toxicity. Levobupivacaine and Bupivacaine have neuroaxial anesthetic equipotent efficacy, and the mean effective dose for motor block is $4.8 \mathrm{mg}$ (4.4 to 5.2). Levobupivacaine has been compared vs. racemic bupivacaine $(4,8$ and $11 \mathrm{mg}$ ) in healthy volunteers finding that the sensory block, motor block and time to complete discharge criteria were similar, concluding that both drugs have a clinical equivalence in the doses used.

\section{Ropivacaine}

Ropivacaine is identified as AL in 1957 by Ekenstam; it was recently introduced into the clinic for epidural and locoregional use. It is the first levoisomeric AL introduced to commerce. It is less soluble than bupivacaine, and is the least toxic in the PPX family, with marked differential blockage. Multiple clinical investigations prove its efficacy and safety when injected into the subarachnoid space, and in some European countries it is approved for intrathecal use.

It is fat soluble, with two thirds the potency of bupivacaine, which theoretically favors a better recovery, making it eligible for outpatient surgery. However, some studies have shown that in equipotent doses with bupivacaine, the recovery period is similar for both drugs. In healthy volunteers it was found to have half the potency of bupivacaine, with a higher incidence of side effects, particularly low back pain, although the possibility of ITRP is $0-1 \%$. Other studies found that $15 \mathrm{mg}$ of intrathecal ropivacaine produced motor block and hemodynamic effects similar to $10 \mathrm{mg}$ of bupivacaine, but with less powerful anesthesia. Ropivacaine $0.5 \%$ hyperbaric $15 \mathrm{mg}$ was superior to bupivacaine $0.5 \%$ hyperbaric 15 mg in outpatients with lower abdomen surgery. When associated with clonidine (ropivacaine $8 \mathrm{mg}+$ clonidine $15 \mu \mathrm{g}$ ), it produces a short-term sensory block, sufficient for outpatient arthroscopies.

For ambulatory surgeries below $\mathrm{T} 10$, one of these three ALs can be used in its isobaric form. When surgical procedures span metamers above T10, it is recommended to use hyperbaric presentations.

Hyperbaric solutions of local anesthetics diffuse in the CSF in a more uniform way than iso or hypobaric solutions, since this diffusion depends more on its baricity than on other factors that modulate the intrathecal diffusion of local isobaric anesthetics such as volume. CSF. Diffusion can be modified by positioning the patient within the first 20 minutes after injecting the hyperbaric anesthetic. Once the maximum level of spinal anesthesia is reached, it is possible to gain a couple of segments with intravenous injection of clonidine at a dose of $3 \mu \mathrm{g} / \mathrm{kg}$. When it comes to brief procedures, it is advisable to decrease the doses of any of these three LAs. It is possible to decrease the doses of these three drugs to $5 \mathrm{mg}$ by adding an adjuvant such as 45 to $75 \mu$ of clonidine, or fentanyl 12.5 to $25 \mu \mathrm{g}$. This combination will decrease the incidence of motor blockage and could prolong the duration of the AL $[10,11]$.

\section{Mepivacaine}

Mepivacaine has been used intrathecally since 1960. It belongs to the PPX family, it has a short onset of action, with intermediate duration and low toxicity. It has a fast liver metabolism and is excreted by the kidneys. Its clinical characteristics are similar to lidocaine, with an approximate power of 1.3: 1. Produces ITRP between 0 and 30\%. Mepivacaine $4 \%$ hyperbaric has been mixed with bupivacaine $0.5 \%$ isobaric and compared with bupivacaine $0.5 \%$ hyperbaric, improving the latency time and anesthetic duration up to 3 hours.

\section{Chloroprocaine}

Chloroprocaine. The short-acting LAs that could be used subarachnoidly to replace lidocaine in this group of outpatients are chloroprocaine and procaine. Both drugs belong to the ester 
group, they are derivatives of paraaminobenzoic acid, with high pKa and poor fat solubility. They are rapidly hydrolyzed by plasma cholinesterases, which limits their systemic toxicity. They have a rapid onset of action and are short in duration. Chloroprocaine has an antagonistic effect on the $\mu$ and $\mathrm{k}$ opioid receptors, which interferes with the administration of neuroaxial opioids.

Eight volunteers were studied, each receiving $40 \mathrm{mg}$ of lidocaine $2 \%$ or $40 \mathrm{mg}$ of chloroprocaine $2 \%$ without preservative, and it was found that the latter produced anesthetic efficacy similar to lidocaine, including the height of the block. The sensory block lasted less with intrathecal chloroprocaine than with lidocaine. No patient treated with chloroprocaine had ITRP, compared to 7 of the 8 lidocaine subjects.

\section{Procaine}

Procaine has been used in the subarachnoid space for brief surgeries since the beginning of the last century, but it was displaced by lidocaine since the latter produces more reliable anesthesia. Its onset of action is slower than chloroprocaine, and its duration of action is between 30 and 60 minutes. The intrathecal dose is 50 to $200 \mathrm{mg}$, of the $10 \%$ solution. It has been shown to have a lower incidence of ITRP than lidocaine ( $6 \%$ vs. $24 \%$ ) but produces more nausea.

In general, the use of adjuvant drugs that allow us to decrease the dose of LA, facilitate prompt recovery, and effective postoperative analgesia is common.

There are numerous spinal receptors that modulate the pain response, however, there are only several useful drugs via the subarachnoid pathway that act as adjuvants. Adrenaline was the adjuvant most used in subarachnoid anesthesia before the arrival of neuroaxial opioids. Its effect has been questioned; Doses of 100 to $300 \mu \mathrm{g}$ added to hyperbaric lidocaine or bupivacaine did not prolong the block time and did delay recovery. Some authors have reported a brief prolongation of the anesthetic effects of lidocaine.

\section{Opioids}

In 1976 Yask and Rudy 64 demonstrated for the first time that intrathecal opioids produced potent and selective analgesia in animals without supraspinal effect. The application of spinal opioids for analgesic purposes was first described in humans in 1977 by Wang. This author reported his research on rats and mentioned in the last paragraph of his article that a clinical study with intrathecal morphine had been done at the Mayo Clinic in patients with stubborn pain secondary to cancer. In this report the author insisted on the importance of treating pain without affecting other neurological functions.

The most studied intrathecal opioids in outpatients are fentanyl and sufentanil. The first is the most widely used and its effects are dose dependent. Doses range from $25 \mu \mathrm{g}$ to $10 \mu \mathrm{g}$, with a tendency to use the smallest dosages. Pruritus is a frequent side effect of intrathecal opioids, which depends on the total dose administered.
It can reach up to $60 \%$ with $10 \mu \mathrm{g}$ of fentanyl, but only $20 \%$ to $30 \%$ requires treatment. The use of intrathecal morphine is not recommended in outpatients since the possibility of urinary retention is high, which can prolong your hospital stay.

\section{Alpha2 agonists}

They have a mechanism of action not mediated by the opioid system. Adrenaline was the first drug in this group to be used intrathecally. Clonidine is an alpha2 agonist that has been used in anesthesiology for various purposes. When injected into the neuroaxial route, it prolongs motor and sensory block, while increasing sedation and can potentiate hypotension secondary to sympathetic block typical of spinal anesthesia. It has been extensively studied via the subarachnoid route, and there are controversies between high doses ( $>150 \mu \mathrm{g})$ and low doses $(<150$ $\mu \mathrm{g})$. Doses of 150, 300 and $450 \mu \mathrm{g}$ demonstrated dose-dependent analgesia.

When a subarachnoid block fails, it can be repeated after 20 minutes, which allows the certainty that the first injection attempt will have no summative effects with the second dose of AL. We must be sure that the first dose reached its maximum effect and bear in mind that the diffusion of ALs can be very slow in some patients. The 20 min wait ensures that most of the local anesthetic that reached the subarachnoid space has been "fixed" in the neural tissue.

Repeated correct injection into the same space, or even better into superior spaces, usually produces adequate subarachnoid block. It is prudent to monitor that the total dose of the injected AL does not exceed the recommended total dose to avoid local neurotoxicity, or anesthetic effects. If possible, it is recommended that another type of LA be injected in the second subarachnoid puncture [12-15].

\section{Acknowledgement}

None.

\section{Conflict of Interest}

No conflict of interest

\section{References}

1. Saikia P, Karim HMR (2020) Letter in response to Xiao et al: ED50 and ED95 of intrathecal bupivacaine with or without phenylephrine. Reg Anesth Pain Med 45(3): 250.

2. Tubog TD, Ramsey VL, Filler L, Bramble RS (2018) Minimum Effective Dose (ED (50) and ED (95)) of Intrathecal Hyperbaric Bupivacaine for Cesarean Delivery: A Systematic Review. AANA J 86(5): 348-360.

3. Siedler G, Kallmünzer B, Schwab S (2019) Dtsch Med Wochenschr 144(19): 1361-1366

4. Lacroix SP, Malaise PR, Degey SV, Deflandre EP (2019) Predictability of the duration of motor blockade induced by unique injection of intrathecal prilocaine-an observational study. J Physiol Pharmacol 70(3).

5. Irwin R, Stanescu S, Buzaianu C, Rademan M, Roddy J, et al. (2020) Quadratus lumborum block for analgesia after caesarean section: a randomised controlled trial. Anaesthesia 75(1): 89-95. 
6. Guay J, Williams SR, Robin F, Ruel M (2019) Effect of Intravenous Dexamethasone on the Regression of Isobaric Bupivacaine Spinal Anesthesia: A Randomized Controlled Trial. Anesth Analg 128(6): e100-e103.

7. Gautam B, Tabdar S, Shrestha U (2018) Comparison of Fentanyl and Dexmedetomidine as Intrathecal Adjuvants to Spinal Anaesthesia for Abdominal Hysterectomy. JNMA J Nepal Med Assoc 56(213): 848-855.

8. Liu L, Qian J, Shen B, Xiao F, Shen H (2019) Intrathecal dexmedetomidine can decrease the $95 \%$ effective dose of bupivacaine in spinal anesthesia for cesarean section: A prospective, double-blinded, randomized study. Medicine 98(9): e14666.

9. Vives R, Fernandez Galinski D, Gordo F, Izquierdo A, Oliva JC, et al. (2019) Effects of bupivacaine or levobupivacaine on cerebral oxygenation during spinal anesthesia in elderly patients undergoing orthopedic surgery for hip fracture: a randomized controlled trial. BMC Anesthesiol 19(1): 17.

10. Park SK, Lee JH, Yoo S, Kim WH, Lim YJ, et al. (2019) Comparison of bupivacaine plus intrathecal fentanyl and bupivacaine alone for spinal anesthesia with intravenous dexmedetomidine sedation: a randomized, double-blind, noninferiority trial. Reg Anesth Pain Med 44(4): 459-465.
11. Hussien RM, Rabie AH (2019) Sequential intrathecal injection of fentanyl and hyperbaric bupivacaine at different rates: ¿does it make a difference? A randomized controlled trial. Korean J Anesthesiol 72(2): 150-155.

12. Xu W, Xiao F, Zhang Y, Liu L, Chang X (2018) ED50 and ED95 of intrathecal hyperbaric ropivacaine for parturients undergoing cesarean section with prophylactic infusion of phenylephrine: A Prospective dose-finding Study. Medicine 97(50): e13727.

13. Kyeong Kim M, Shin J, Choi JH, Yong Kang H (2018) Low-dose combined spinal-epidural anesthesia for a patient with a giant hiatal hernia who underwent urological surgery. J Int Med Res 46(10): 4354-4359.

14. Xiao F, Drzymalski D, Liu L, Zhang Y, Wang L, et al. (2018) Comparison of the ED50and ED95 of Intrathecal Bupivacaine in Parturients Undergoing Cesarean Delivery With or Without Prophylactic Phenylephrine Infusion: A Prospective, Double-Blind Study. Reg Anesth Pain Med 43(8): 885-889.

15. Liu J, Wang Y, Ma W (2018) Shivering prevention and treatment during cesarean delivery under neuraxial anesthesia: a systematic review. Minerva Anestesiol 84(12): 1393-1405. 